\title{
CONTINUITY OF CERTAIN CONNECTED FUNCTIONS AND MULTIFUNCTIONS
}

\author{
MELVIN R. HAGAN ${ }^{1}$
}

\begin{abstract}
In this paper it is proved that if $X$ is a 1st countable, locally connected, $T_{1}$-space and $Y$ is a $\sigma$-coherent, sequentially compact $T_{1}$-space, then any nonmingled connectedness preserving multifunction from $X$ onto $Y$ with closed point values and connected inverse point values is upper semicontinuous. It follows that any monotone, connected, single-valued function from $X$ onto $Y$ is continuous. Let $X$ be as above and let $Y$ be a sequentially compact $T_{1}$-space with the property that if a descending sequence of connected sets has a nondegenerate intersection, then this intersection must contain at least three points. If $f$ is a monotone connected single-valued function from $X$ onto $Y$, then $f$ is continuous. An example of a noncontinuous monotone connected function from a locally connected metric continuum onto an hereditarily locally connected metric continuum is given.
\end{abstract}

In [1] and [2] conditions are given under which an open monotone connected function is continuous. This paper is concerned with conditions under which a monotone connected function is continuous. As Example 2 below shows, a monotone connected function from an hereditarily locally connected metric continuum onto a nonlocally connected metric continuum is not necessarily continuous, and Example 3 shows that a monotone connected function from a locally connected metric continuum onto an hereditarily locally connected metric continuum is not necessarily continuous. It is an open question as to whether or not such a function is continuous if both the domain and range are hereditarily locally connected metric continua.

Some definitions will now be recalled. A multifunction $F: X \rightarrow Y$ is upper semicontinuous at a point $p \in X$ if for any open set $V \subset Y$, with $F(p) \subset V$, there is an open set $U \subset X$, with $p \in U$, such that $F(U) \subset V$, and $F$ is nonmingled provided for any $p, q \in X$, either $F(p)=F(q)$ or $F(p) \cap F(q)=\varnothing$.

Received by the editors August 25, 1972.

AMS (MOS) subject classifications (1970). Primary 54C10, 54C60; Secondary 54F20, $54 \mathrm{~F} 50$.

Key words and phrases. Upper semicontinuous multifunction, connectedness preserving function, monotone function, locally connected, $\sigma$-coherent, hereditarily locally connected continuum.

1 Research supported by North Texas State University Faculty Research Grant No. 34590.

(c) American Mathematical Society 1974 
See [6] for other terminology and properties of multifunctions. A singlevalued function is monotone if point inverses are connected and is connected if the function preserves connectedness. This same terminology will be used for multifunctions also. A space $Y$ is $\sigma$-coherent provided any descending sequence of connected sets has a connected intersection. Finally, $\lim \sup A_{n}$ will denote the upper limit of a sequence $\left\{A_{n}\right\}$ of sets as defined on p. 337 of [3].

THEOREM 1. Let $X$ be a 1 st countable, locally connected $T_{1}$-space and $Y$ a $\sigma$-coherent, sequentially compact $T_{1}$-space. If $F$ is a nonmingled connected multifunction from $X$ onto $Y$ with closed point values, such that $F^{-1}$ has connected point values, then $F$ is upper semicontinuous.

Proof. Suppose $F$ is not upper semicontinuous at the point $p \in X$. Then there is an open set $V \subset Y$ such that $F(p) \subset V$, but for any open set $U \subset X$, with $p \in U$, there is some $x \in U$ such that $F(x)$ is not contained in $V$. Let $\left\{U_{n}\right\}$ be a countable base at $p$ consisting of open connected sets with $U_{n+1} \subset U_{n}$ for all $n$. For each $n$, let $p_{n} \in U_{n}$ such that $F\left(p_{n}\right)$ is not contained in $V$, and let $A_{n}=(Y-V) \cap F\left(p_{n}\right)$. Then $\left\{A_{n}\right\}$ is a sequence of sets all lying in the closed set $Y-V$. Since $Y$ is sequentially compact, there is a point $q \in(Y-V) \cap \lim \sup A_{n}$. If $q \in F\left(p_{n}\right)$ for all but finitely many $n$, then it can be assumed that $q \in F\left(p_{n}\right)$ for all $n$. Thus, $F\left(p_{1}\right)=F\left(p_{n}\right)$ for all $n$, since $F$ is nonmingled. Therefore, $F\left(p_{1}\right) \subset F\left(U_{n}\right)$ for all $n$, which implies that $q \in K=$ $\bigcap_{n=1}^{\infty} F\left(U_{n}\right)$. Also, $F(p) \subset V$ and $q \in(Y-V)$. Hence, $F(p)$ and $q$ are separated in $K$ since $F(p) \subset K$. But $K$ is connected since $Y$ is $\sigma$-coherent. Therefore, there is a point $y$ in $K-(F(p) \cup q)$. Since $y \in F\left(U_{n}\right)$ for all $n$, $F^{-1}(y) \cap U_{n} \neq \varnothing$ for all $n$. Therefore $p$ is a limit point of $F^{-1}(y)$. But by Corollary $\mathrm{D}_{2}$ of [6], $F^{-1}(y)$ is closed. Hence, $p \in F^{-1}(y)$. This implies $y \in F(p)$, which is a contradiction. Thus, it must be the case that $q \notin F\left(p_{n}\right)$ for infinitely many $n$. Now $F\left(p_{j}\right) \subset F\left(U_{n}\right)$ for all $j \geqq n$, and every neighborhood of $q$ intersects $F\left(p_{j}\right)$ for infinitely many $j$. Thus, $q$ is a limit point of $F\left(U_{n}\right)$ for every $n$. Therefore, $\left\{F\left(U_{n}\right) \cup q\right\}$ is a descending sequence of connected sets, and by hypothesis $K=\bigcap_{n=1}^{\infty}\left(F\left(U_{n}\right) \cup q\right)$ is connected. But again, $F(p)$ and $q$ are separated in $K$ and therefore there is a point $y$ in $K-(F(p) \cup q)$. This leads to the same contradiction as before. Hence, $F$ must be upper semicontinuous.

COROLlary. If $X$ and $Y$ are as in Theorem 1 and $f$ is a monotone, connected, single-valued function from $X$ onto $Y$, then $f$ is continuous.

Proof. A single-valued function is nonmingled, and since $Y$ is $T_{1}, f$ has closed point values. Also, $f$ monotone means $f^{-1}$ has connected point values. 
THEOREM 2. Let $X$ be as in Theorem 1 and let $Y$ be a sequentially compact $T_{1}$-space with the property that if a descending sequence of connected sets has a nondegenerate intersection, then this intersection must contain at least three points. If $f$ is a monotone connected single-valued function from $X$ onto $Y$, then $f$ is continuous.

ProOF. The proof proceeds exactly as in the proof of Theorem 1. The set $K$ has at least two points $f(p)$ and $q$, so by hypothesis must contain a third point $y$ distinct from $f(p)$ and $q$. This leads to the same contradiction as in the proof of Theorem 1. Thus, $f$ must be upper semicontinuous. But this is equivalent to continuity since $f$ is single-valued.

The following example shows that not every hereditarily locally connected metric continuum has the property given in the hypothesis of Theorem 2, that if a descending sequence of connected sets has a nondegenerate intersection, then the intersection has at least three points.

Example 1. This is a modification of an example given in [4, p. 284]. In the plane let $C_{n k}$ denote the semicircle given by

$$
\left(x-(2 k-1) / 2^{n}\right)^{2}+y^{2}=1 / 4^{n}, \quad y \geqq 0 .
$$

Denote by $L_{n k}$ the straight line segment given by $x=(2 k-1) / 2^{n}, 0 \leqq y \leqq$ $1 / 2^{n}$. Let $Q_{n k}$ denote the semicircle given by

$$
\left(x-(2 k-1) /\left(2 \cdot 3^{n}\right)\right)^{2}+y^{2}=1 /\left(4 \cdot 9^{n}\right), \quad y \leqq 0 .
$$

Denote by $R_{n k}$ the straight line segment given by

$$
x=(2 k-1) /\left(2 \cdot 3^{n}\right), \quad-(2 k-1) /\left(2 \cdot 3^{n}\right) \leqq y \leqq 0 .
$$

Let $H_{n}$ denote the union of all the $C_{n k}$ and $L_{n k}, 1 \leqq k \leqq 2^{n-1}$, and denote by $K_{n}$ the union of all the $Q_{n k}$ and $R_{n k}, 1 \leqq k \leqq 3^{n}$. Finally, let $X$ denote the union of all the $H_{n}$ and all the $K_{n}, n$ varying over all positive integers, along with the interval $I=[0,1]$. Then $X$ is an hereditarily locally connected continuum. Let $D$ denote the set of end points of all upper semicircles $C_{n k}$ and $T$ the set of end points of all lower semicircles $Q_{n k}$, and let $p=(0,0)$ and $q=(1,0)$. Let $U_{1}=(X-I) \cup D \cup T$ and for $n \geqq 1$, let $U_{n+1}=$ $\left(U_{n}-\left(H_{n} \cup K_{n}\right)\right) \cup\{p, q\}$. Then $\left\{U_{n}\right\}$ is a descending sequence of connected sets whose intersection consists of just the points $p$ and $q$.

In the following example, an hereditarily locally connected $\sigma$-coherent continuum is mapped by a noncontinuous one-to-one connected function $f$ onto a nonlocally connected, 1 st countable continuum. The function $f^{-1}$ is also connected and noncontinuous. Since all the hypotheses of Theorem 1 is satisfied for $f^{-1}$ except local connectedness of the domain of $f^{-1}$, this property is necessary in Theorem 1 . 
EXAMPLE 2. Choose a polar coordinate system on the plane and for each positive integer $n$, let $L_{n}$ denote the segment $\{(r,(\pi / 2) / n) \mid 0 \leqq r \leqq 1 / n\}$. Let $X=\bigcup_{n=1}^{\infty} L_{n}$. Now choose a rectangular coordinate system on the plane with the same origin, and for each positive integer $n$, let $S_{n}$ denote the segment $\{(x, y) \mid 0 \leqq x \leqq 1, y=x / n\}$, and $S_{0}$ the segment $\{(x, 0) \mid 0 \leqq x \leqq 1\}$. Then $Y=\bigcup_{n=0}^{\infty} S_{n}$ is a nonlocally connected non- $\sigma$-coherent metric continuum. Define a function $f$ from $X$ onto $Y$ as follows: For each $n \geqq 1$, let $p_{n}=(1 / n,(\pi / 2) / n)$ in polar coordinates, and $q_{n}=(1,1 / n)$ in rectangular coordinates. Let $p_{0}=(0,0)$ and $q_{0}=(1,0)$. Let $f$ be the function that takes $L_{n}$ linearly onto $S_{n-1}$ such that $f\left(p_{0}\right)=p_{0}$ and $f\left(p_{n}\right)=q_{n-1}, n \geqq 1$. Then $f$ is one-to-one, connected, and not continuous at $p_{0}$. Also, the function $f^{-1}$ taking $Y$ onto $X$ satisfies all the hypothesis of Theorem 1 except that the domain $Y$ is not locally connected, and $f^{-1}$ is not continuous at $q_{0}$. Thus, local connectedness of the domain space is necessary in Theorem 1.

Finally, the following is an example of a noncontinuous, monotone, connected function from a locally connected metric continuum onto an hereditarily locally connected metric continuum.

EXAMPLE 3. Choose a rectangular coordinate system on Euclidean 3space $E_{3}$ and let $p=(0,0,0)$ and $q=(1,0,0)$. Also, choose a spherical coordinate system, as defined on p. 355 of [5], with origin $p$. Thus $q=$ $(1,0, \pi / 2)$ in spherical coordinates. For each $n$, let

$$
p_{n}=(1 / n, \pi / 2, \pi /(2 n)) \text { and } q_{n}=\left(\left(2^{n}-1\right) / 2^{n}, 0, \pi / 2\right)
$$

in spherical coordinates. If $a$ and $b$ are end points of a straight line segment, let $a b$ denote the ordered segment from $a$ to $b$. Let $P_{n}$ denote the plane determined by the three points $p, p_{n}, q_{n}, L_{n}$ the ordered segment from $p_{n}$ to $q_{n}$, and $S_{n}$ the ordered segment from $p$ to $p_{n}$.

Define inductively a sequence of finite ordered subsets of $p q$ as follows: Let $H_{2}=\left\{x_{21}, x_{22}\right\}$, where $x_{21}$ and $x_{22}$ are the mid points, respectively, of the segments $p q_{1}$ and $q_{1} q_{2}$. Let $H_{3}=\left\{x_{31}, x_{32}, x_{33}, x_{34}, x_{35}\right\}$, where these points are, respectively, the mid points of the segments $p x_{21}, x_{21} q_{1}, q_{1} x_{22}$, $x_{22} q_{2}, q_{2} q_{3}$. Assuming $H_{n-1}$ has been defined, let $H_{n}=\left\{x_{n 1}, \cdots, x_{n k_{n}}\right\}$, where the $x_{n i}$ are the mid points of the ordered collection of segments of $p q_{n}$ determined by the points of $H_{n-1}$. The order of the listing in $H_{n}$ is by increasing distance from $p$, where $x_{n 1}$ is the mid point of the segment $p x_{n-1.1}$ and $x_{n k_{n}}$ is the mid point of the segment $q_{n-1} q_{n}$. Note that the union of the $H_{n}$ 's is a countable dense subset of $p q$.

For each $n \geqq 2$ and each $j, 1 \leqq j \leqq k_{n}$, let $L_{n j}$ denote the line segment parallel to $L_{n}$ with one end point at $x_{n j}$ and the other end point, denoted by $s_{n j}$, on the segment $S_{n}$. Thus, $\left\{s_{n 1}, \cdots, s_{n k_{n}}\right\}$ is a finite subset of $S_{n}$ and each $s_{n j}$ is joined to the corresponding $x_{n j}$ by the segment $L_{n j}$ lying in the plane $P_{n}$ and parallel to $L_{n}$. 
Let $K_{n}=\left(\bigcup_{i=2}^{n} H_{i}\right) \cup\left\{q_{1}, \cdots, q_{n-1}\right\}$. For each point $y \in K_{n}$, let $L_{y}$ denote the segment parallel to $S_{n}$ with one end point at $y$ and the other end point on $L_{n}$. Thus, each $L_{y}$ lies in the plane $P_{n}$. Let $Q_{1}=p q \cup p p_{1} \cup p_{1} q_{1}$, and for $n \geqq 2$, let $Q_{n}$ denote the union of the segments $p q, S_{n}, L_{n}, L_{y}, y \in K_{n}$, and $L_{n j}, 1 \leqq j \leqq k_{n}$. Then $X=\bigcup_{n=1}^{\infty} Q_{n}$ is a locally connected continuum.

REMARK. Let $R_{n}$ denote the plane given by $z=y / n$ in rectangular coordinates, where $n$ is a positive integer. It is to be understood that any arc of a circle subsequently described with end points on $p q$ and lying in $R_{n}$ is to have altitude less that one-half of the minimum of the altitudes of all such previously described arcs and is disjoint from all such arcs except possibly at some end points.

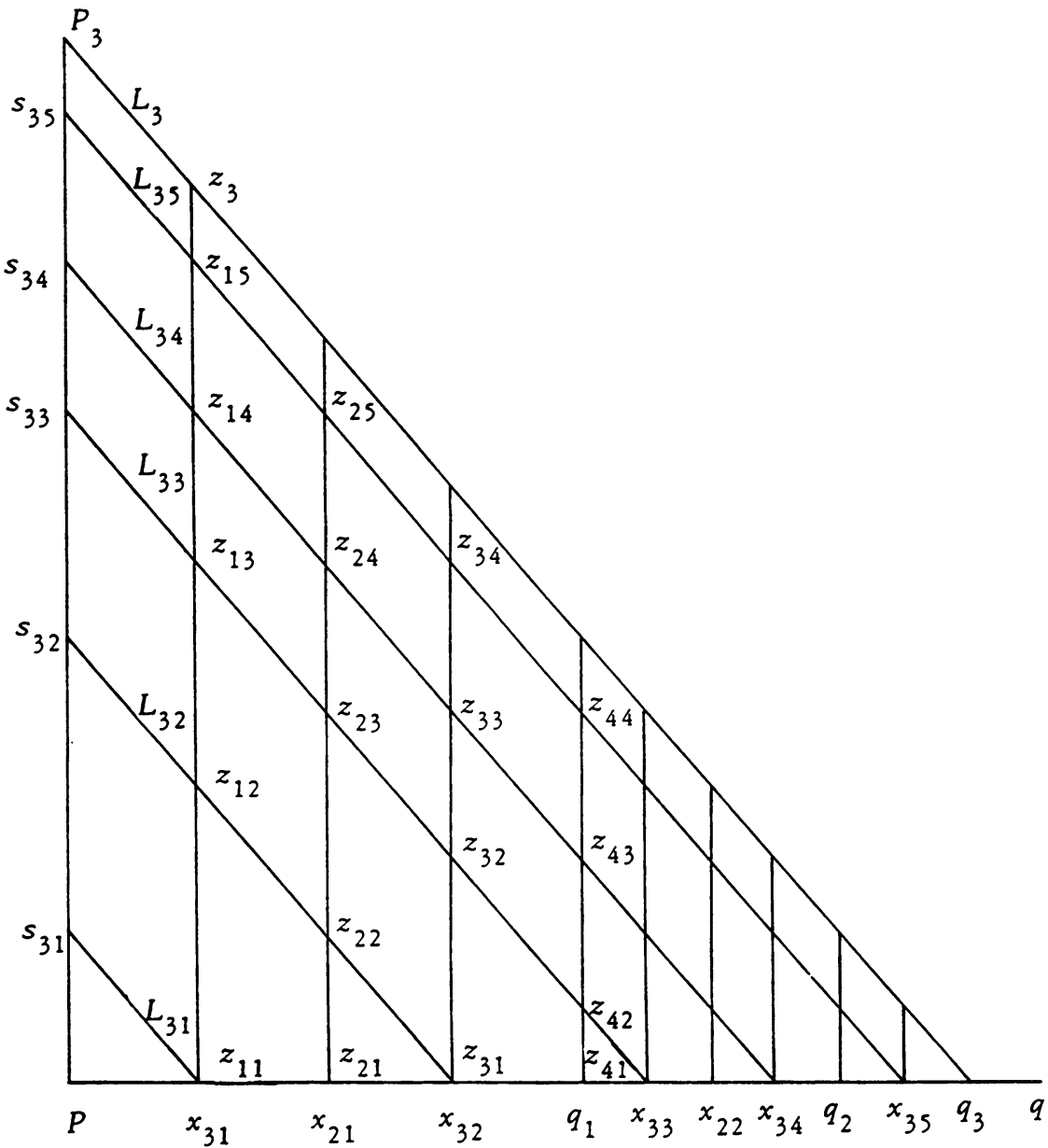

Figure 1 
Define a function $f$ on $X$ into $E_{3}$ as follows: It will be sufficient to define $f$ on each $Q_{n}$. Note that $Q_{1}=p q \cup p p_{1} \cup p_{1} q_{1}$. The function $f$ will be the identity function on $p q$. Let $f$ take $p p_{1}$ homeomorphically onto an arc of a circle of radius one-half the distance from $p$ to $q_{1}$ lying in $R_{1}$ with end points $p$ and $q_{1}$ with $f\left(p_{1}\right)=q_{1}$, and let $f\left(p_{1} q_{1}\right)=q_{1}$. The function $f$ will now be defined on $Q_{3}$ and it will be clear that the same process can be used on any $Q_{n}$. The set $K_{3}$ consists of the points $x_{31}, x_{21}, x_{32}, q_{1}, x_{33}, x_{22}, x_{34}, q_{2}$, $x_{35}$, and $Q_{3}$ is

$$
p q \cup S_{3} \cup L_{3} \cup\left(\bigcup_{i=1}^{5} L_{3 i}\right) \cup\left(\cup\left\{L_{y} \mid y \in K_{3}\right\}\right) .
$$

Define $f$ on $s_{3}$, which is $p s_{31} \cup s_{31} s_{32} \cup s_{32} s_{33} \cup s_{33} s_{34} \cup s_{34} s_{35} \cup s_{35} p_{3}$, as follows: Let $f$ take $p s_{31}$ homeomorphically onto an arc of a circle lying in $R_{3}$ where the arc has end points $p$ and $x_{31}$ and altitude one-half the distance from $p$ to $x_{31}$, and $f\left(s_{31}\right)=x_{31}$. Let $f$ take $s_{31} s_{32}$ homeomorphically onto an arc of a circle lying in $R_{3}$ where the arc has end points $x_{31}, x_{32}$ and is subject to the conditions in the above remark, and $f\left(s_{32}\right)=x_{32}$. Map segments $s_{32} s_{33}, s_{33} s_{34}$, and $s_{34} s_{35}$ in a similar manner. Let $f$ take $s_{35} p_{3}$ homeomorphically onto an arc of a circle lying in $R_{3}$ where the arc has end points $x_{35}, q_{3}$ and subject to the conditions in the above remark, and $f\left(s_{35}\right)=x_{35}$ and $f\left(p_{3}\right)=q_{3}$. Let $f\left(L_{3}\right)=q_{3}$ and $f\left(L_{3 i}\right)=x_{3 i}, 1 \leqq i \leqq 5$.

For $y=x_{31} \in K_{3}, L_{y}$ intersects $L_{3 j}$ at, say $z_{1 j}, 1 \leqq j \leqq 5$, where $z_{11}=x_{31}$, and $L_{y}$ intersects $L_{3}$ at, say $z_{3}$. Map $x_{31} z_{12}$ homeomorphically onto an arc of a circle lying in $R_{3}$ satisfying the conditions in the above remark, with end points $x_{31}, x_{32}$, where $f\left(z_{12}\right)=x_{32}$. Let $f$ take $z_{12} z_{13}$ homeomorphically onto an appropriate arc of a circle lying in $R_{3}$ with end points $x_{32}, x_{33}$, where $f\left(z_{13}\right)=x_{33}$. Map $z_{13} z_{14}$ homeomorphically onto an appropriate arc

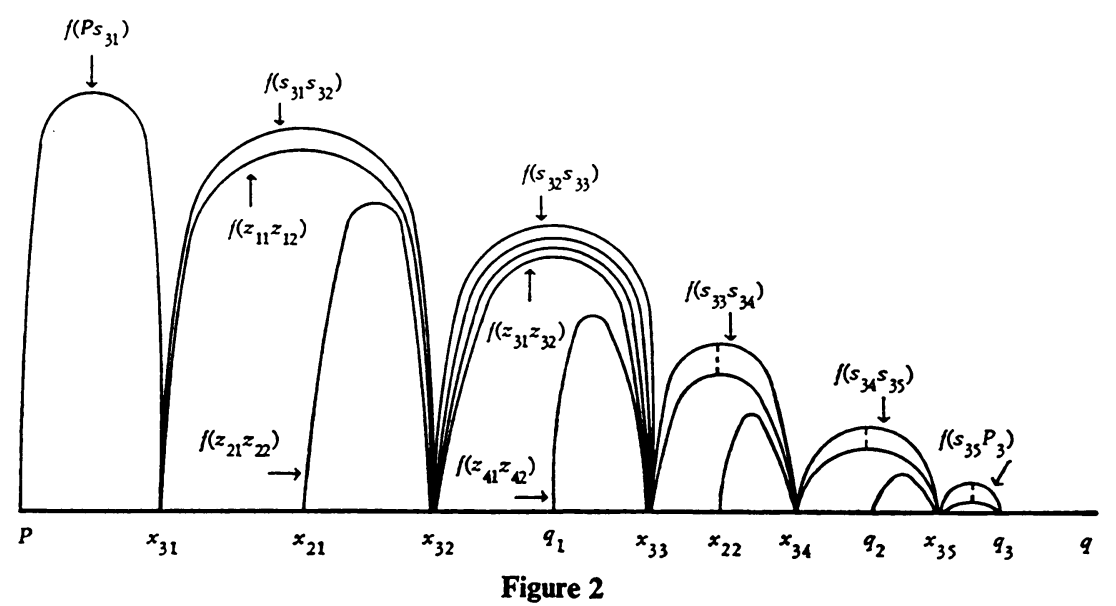


of a circle lying in $R_{3}$ with end points $x_{33}, x_{34}$, where $f\left(z_{14}\right)=x_{34}$. Map $z_{14} z_{15}$ in a similar manner. Let $f$ take $z_{15} z_{3}$ homeomorphically onto an appropriate arc of a circle lying in $R_{3}$ with end points $x_{35}, q_{3}$, where $f\left(z_{15}\right)=$ $x_{35}$ and $f\left(z_{3}\right)=q_{3}$. This defines $f$ on $L_{y}$, where $y=x_{31}$. Define $f$ similarly on each $L_{y}, y \in K_{3}$, where the arcs chosen are subject to the conditions in the above remark. The function is now defined on $Q_{3}$. The set $Q_{3}$ and its image $f\left(Q_{3}\right)$ are displayed in Figures 1 and 2, respectively. In Figure 2 the curves are supposed to represent circular arcs. Define $f$ in a similar manner on all the $Q_{n}$, where the initial arcs are to be chosen in $R_{n}$ having radius one-half the distance from $p$ to $x_{n 1}$, and subject to the conditions in the above remark.

The resulting function $f$ is monotone since the inverse of a point is either a point or a segment, $f$ maps connected sets onto connected sets, $f(X)$ is a hereditarily locally connected continuum in $E_{3}$, but $f$ is not continuous at $p$ since the sequence $\left\{p_{n}\right\}$ converges to $p$ and the sequence $\left\{f\left(p_{n}\right)\right\}$ converges to $q \neq f(p)$.

\section{BIBLIOGRAPHY}

1. M. R. Hagan, A note on connected and peripherally continuous functions, Proc. Amer. Math. Soc. 26 (1970), 219-223. MR 41 \#7647.

2. - Conditions for continuity of certain open monotone functions, Proc. Amer. Math. Soc. 30 (1971), 175-178. MR 43 \#5500.

3. K. Kuratowski, Topologie. Vol. I, 2nd ed., Monografie Mat., Tom 20, WarszawaWroclaw, 1948; English transl., New ed., rev. and aug., Academic Press, New York; PWN, Warsaw, 1966. MR 10, 389; 36 \#840.

4. - Topologie. Vol. II, 3rd ed., Monografie Mat., Tom 21, PWN, Warsaw, 1961; English transl., Academic Press, New York; PWN, Warsaw, 1968. MR 24 \#A2958; 41 \#4467.

5. A. E. Taylor, Advanced calculus, Ginn, Boston, Mass., 1955.

6. G. T. Whyburn, Continuity of multifunctions, Proc. Nat. Acad. Sci. U.S.A.54(1965), 1494-1501. MR 32 \#6423.

Department of Mathematics, North Texas State University, Denton, Texas 76203 